\title{
Comparison and Evaluation of Prelens Tear Film Stability by Different Noninvasive in vivo Methods
}

This article was published in the following Dove Press journal:

Clinical Ophthalmology

\author{
Takashi Itokawa (1D) ${ }^{1,2}$ \\ Takashi Suzuki ${ }^{2,3}$ \\ Hiroko Iwashita (ID) \\ Yuichi Hori ${ }^{1,2}$ \\ 'Department of Ophthalmology, Toho \\ University Graduate School of Medicine, \\ Tokyo, Japan; ${ }^{2}$ Department of \\ Ophthalmology, School of Medicine, \\ Toho University, Tokyo, Japan; ${ }^{3}$ Ishizuchi \\ Eye Clinic, Niihama, Ehime, Japan
}

\begin{abstract}
Purpose: Prelens tear film stability of soft contact lens (SCL) play an important role for contact lens discomfort. In this study, we investigated the association between two types of noninvasive methods and evaluated the tear film stability with SCL using the methods.

Patients and Methods: In experiment 1, images of ring mire were recorded with a keratograph after focusing the pigment located at the front or back of the SCL. Interferometry and videokeratoscopy were used for the assessment of tear film stability in the right eye of 10 women, with two different cosmetic daily disposable SCLs: polymacon and etafilcon A with polyvinylpyrrolidone. Time to first distortion by noninvasive keratograph break up time (NIKBUT-first) was compared to noninvasive interferometry break up time (NIBUT). In experiment 2, ten normal females wore two different daily disposable SCLs: samfilcon A and narafilcon A. NIKBUT-first and NIBUT were compared between the lenses after 8 hours of SCL wearing.

Results: In experiment 1 , NIBUT-first without SCL was significantly correlated to NIBUT without SCL ( $\mathrm{r}=0.445, \mathrm{P}=0.0488$, Pearson's correlation coefficients). However, NIKBUTfirst with SCL was not significantly correlated with NIBUT with SCL. In experiment 2, although NIKBUT-first was not significantly different between SCLs, samfilcon A had significantly longer NIBUT than narafilcon A $(\mathrm{P}=0.0315$, paired $t$-test).

Conclusion: NIKBUT-first with SCL could be related to tear film stability between the lens and the corneal surface, but not to prelens tear film stability. NIIBUT could be a suitable method to evaluate prelens tear stability.
\end{abstract}

Keywords: tear film stability, wettability, tear meniscus height, soft contact lens, silicone hydrogel lens

\section{Introduction}

There are more than 140 million contact lens wearers worldwide. ${ }^{1}$ Soft contact lenses (SCLs) are not only used for refractive correction but also for therapeutic, ${ }^{2}$ cosmetic, ${ }^{3}$ and diagnostic ${ }^{4}$ applications. It is well known that dry eye symptoms do not develop in the eyes without SCLs but only appear when wearing SCLs. These symptoms have been described by several terms, such as contact lens-related complications, ${ }^{5}$ dryness, ${ }^{6,7}$ and discomfort. ${ }^{7}$ The Tear Film and Ocular Surface (TFOS) International workshop unified these complications as Contact Lens Discomfort (CLD). ${ }^{8}$ Although the etiology of CLD may include various factors, two factors, namely contact lens material and environment could be very important. ${ }^{8}$ In contact lens materials, prelens tear film stability plays an important role in relation to the friction between the SCL surface and the lid wiper and/or bulbar conjunctiva.
Correspondence: Takashi Suzuki Department of Ophthalmology, School of Medicine, Toho University, 6-II-I, Omori-Nishi, Ota-Ku, Tokyo 143-854I, Japan

Tel +8I-3-3762-4I5I

Fax +8I-3-3298-0030

Email t-suzuki@ishizuchi-eye-clinic.jp 
The prelens tear film stability of SCLs has been measured in vivo using videokeratoscopy, ${ }^{9,10}$ interferometry, ${ }^{11-13}$ evaporation rate, ${ }^{14}$ and temperature, ${ }^{13,15}$ and as contact angle (CA) wettability ${ }^{16}$ in vitro. One device used to measure tear film stability is DR-1 $\alpha$ (Kowa, Tokyo, Japan), which is based on interferometry. ${ }^{11}$ Maruyama et al reported that tear film stability and tear film thickness decrease when the ambient temperature and relative humidity are low. ${ }^{11}$ The difference between tear film stability in four types of SCLs has also been evaluated using DR-1 $\alpha$, tear film thickness, and ocular surface temperature changes. ${ }^{13}$ Kaido et al reported that tear film stability measured with DR-1 $\alpha$ was better in asymptomatic subjects than that of symptomatic subjects. ${ }^{17}$ Interferometry measured by DR-1 $\alpha$ has been considered to be a useful instrument for evaluating tear film stability not only in the cornea but also with SCL. Yokoi and Georgiev reported five types of breakup patterns (BUPs) on the cornea, as a novel evaluation method differentiating between various pathophysiologies involved in dry eye. ${ }^{18}$ Additionally, Yokoi et al reported six types of BUPs on the cornea with SCL. ${ }^{19}$

Videokeratoscopy projecting ring mire on the cornea has been widely used as a method for evaluating tear film stability. Among the videokeratoscopy instruments, the Oculus Keratograph 5M (Oculus, Wetzlar, Germany) can not only assess topography ${ }^{20}$ but also parameters related to tear stability, including tear breakup time (TBUT), ${ }^{21}$ tear meniscus height (TMH) ${ }^{14}$ meibography, ${ }^{22}$ and conjunctival redness. ${ }^{23}$ The Keratograph captures images at 32 frames per second and can track tear film integrity. Analysis of 22,000 data points per frame shows points of breakups on a grid mapping the corneal surface, and provides two noninvasive TBUT: noninvasive Keratograph BUT (NIKBUT)-first (the time taken for the first appearance of a break in the tear film) and NIKBUTAverage (average of the time taken to breakup in all the regions monitored over the duration). ${ }^{21}$ Since NIKBUT installed in the machine is automatically measured, the data cannot be influenced by the skill of the investigator. Recently, keratography has begun to be used for the evaluation of prelens tear film stability of SCL. Montero et al reported that repeatability of NIKBUT after wearing SCL decreases in comparison to that of before wearing SCL. ${ }^{24}$ They also reported that NIKBUT was not significantly different among three types of silicon hydrogel contact lenses. ${ }^{25}$ Mousavi et al reported that the evaluation of the prescribed SCL using conventional methods does not always correspond to the assessment of the tear film stability by NIKBUT. ${ }^{26}$ Although the evaluation of prelens tear film stability of SCL plays an important role in identifying CLD, little is known about the comparison of prelens tear film stability measured using different methods. The aim of this study was to compare the measurements of prelens tear film stability of different SCLs using two different methods.

\section{Patients and Methods}

\section{Subjects}

This study was designed as a prospective, crossover, randomized, double masked study and was conducted at the Ishizuchi eye clinic. We enrolled 10 normal female subjects (mean age $\pm \mathrm{SD}, 28.1 \pm 6.1$ years) in experiment 1 and 10 normal female subjects in experiment 2 (35.5 \pm 3.6 years). Measurements were performed in the right eyes of the subjects between 3:00 and 6:00 PM. Room temperature and humidity was maintained at $23-25^{\circ} \mathrm{C}$ and $30-40 \%$. We included habitual SCL wearers who did not complain of dry eye symptoms, such as pain, irritation, dryness, and visual disturbances with bare eyes and who had been using SCLs for more than 5 days a week. The exclusion criteria were subjects who had a history of allergic keratoconjunctivitis, dry eye, or ocular surgery. The diagnosis of dry eye was based on the Asia Dry Eye Society diagnostic criteria, which has defined dry eye as a multifactorial disease with unstable tear film and a tear breakup time of less than 5 seconds. ${ }^{27}$ This study was conducted at Ishizuchi eye clinic and objective evaluation and data analysis was performed by authors. That's why this study need to be approved by institutional Review Board (IRB) in Ishizuchi eye clinic. However due not to have IRB in the eye clinic, this study was approved by the IRB of River Side Clinic ( $\# R S C-1803 R B 01)$ and adhered to the tenets of the Declaration of Helsinki. Informed consent was obtained from the patients after explaining the nature and possible consequences of the study. This study was registered in the University Hospital Medical Information Network (UMIN) (Registry No. UMIN000032503).

We determined the sample size based on our previous study, which resulted in a NIBUT of 2.97 and 6.50 seconds in two different types of silicone hydrogel SCLs. ${ }^{13,28}$ This indicated that at least eight people were required for this study design ( $\alpha=0.05$, power $80 \%$ ).

\section{Measurement of Tear Film Stability by Interferometry}

A tear film interferometer (DR-1 $\alpha$ ) with a low magnification $(7.2 \mathrm{~mm} \times 8.0 \mathrm{~mm})$ was used to assess the breakup 
patterns (BUP) and noninvasive interferometry tear BUT (NIBUT) with and without SCLs. ${ }^{11,13,18,19}$ The subjects were asked to blink naturally and then keep their eyes open for 20 seconds. In the subjects with no appearance of a breakup with and without SCLs during the 20 seconds observation period, the NIBUT was recorded as $20 \mathrm{sec}-$ onds. The BUP with SCLs was classified into six patterns similar to a previous report by Yokoi et al. ${ }^{19}$ Area break (AB), Line break (LB), Thin aqueous layer break (TALB), Spot break (SB), Dimple break (DB), and Random break (RB)). These BUPs with SCL were confirmed through the agreement of the evaluators (TI, TS). The mechanism of each BUP has been published previously. ${ }^{18,19}$ Briefly, AB occurs when there is no aqueous tear on the SCL surface; $\mathrm{LB}$, when streak line shape near the lower SCL because of aqueous tear deficient and suction effect on the aqueous tear from the lower tear meniscus; TALB, when colorful interference fringes immediately after eye opening when there is no lipid layer on the SCL surface due to thin aqueous tear; SB, when the wettability of the SCL surface is decreased and observed spot shape; DB, when streak line shape around the central SCL because of impaired wettability of the SCL surface; and RB, after the complete establishment of tear film and the result of evaporation of aqueous tear. Therefore, AB, LB, and TALB were defined as aqueous deficient types. SB and DB were defined as decreased wettability types. RB was defined as increased evaporation type.

\section{Measurement of Tear Film Stability by Videokeratoscopy}

The NIKBUT was evaluated with and without SCL for 20 seconds using the Keratograph $5 \mathrm{M}$. It was measured automatically after two consecutive natural blinks. ${ }^{21}$ The time from immediate eye opening to the appearance of the first breakup was set as NIKBUT-first. The average time during eye opening was set as NIKBUT-average. The TMH was calculated from the captured image in the lower tear meniscus by the same machine. ${ }^{14}$

\section{Measurement of Contact Angle}

CA was measured using the sessile drop technique by the CA analyzer (Drop Master DMs-401, Kyowa Interface Science Co., Ltd., Nobitome Niiza, Japan). ${ }^{29}$ Five new samples of each type of SCLs were evaluated using saline. After retrieval from the blister pack, the SCLs were carefully grasped at the edge using forceps and were carefully contacted with a cloth (TechniCloth TX604, Texwipe, Kernersville, NC) only at the edge to remove the excess liquid. The SCLs were placed on the lens holder and $1 \mu \mathrm{L}$ of saline was dropped on the lens surface. Digital pictures were recorded at a rate of $70 \mathrm{frames} / \mathrm{second}$. The CAs were calculated automatically using the $\theta / 2$ method with the FAMAS Interface Measurement and Analysis System (Kyowa Interface Science Co., Ltd.). We evaluated the data at 0.1 seconds after the saline came into contact with the SCLs.

\section{Measurement of Functional Visual Acuity (FVA)}

We used the FVA measurement system (AS-28, KOWA, Aichi, Japan), previously reported by Kaido et al to measure the FVA. ${ }^{30,31}$ FVA was measured monocularly with best spectacle correction under photopic conditions and natural blinking during a 60 -second period. The outcomes assessed were the baseline visual acuity (VA), FVA, visual maintenance ratio (VMR), and blink frequency. The baseline VA was defined as the standard best-corrected VA. FVA was defined as the mean value of time-wise changes in VA for 60 seconds. The VMR was defined as the FVA value divided by the baseline VA. The blink frequency was defined as the total number of blinks (frequency/minute) for 60 seconds.

\section{Measurement of Subjective Symptoms}

J-CLDEQ-8, the Japanese version of 8-item Contact Lens Dry Eye Questionnaire was used to compare symptoms among the SCL users. ${ }^{32}$ It consists of eight questions ie, discomfort (frequency and grade), dryness (frequency and grade), blurred vision (frequency and grade), desire of closing the eyes, and removing the SCLs from the eyes. The total score was set at 37 points.

\section{Study Protocol}

\section{Experiment I}

This experiment investigated the association between two different methods, ie, interferometry and videokeratoscopy. First, to identify the area of the SCL to focus on, when using the Keratograph, a single subject was asked to wear two types of cosmetic SCLs, ie, polymacon with pigment at the front surface of the lens (Luxury 1-Day; Amijes Co. Ltd., Tokyo, Japan) and polymacon with pigment at the back surface of the lens (Ever Color 1-Day Decolog; Aisei Co. Ltd., Osaka, Japan). We measured 
pigment location by anterior segment optical coherence tomography (AS-OCT; Anterion, Heidelberg Engineering $\mathrm{GmbH}$, Dossenheim, Germany). To assess the sharpness of the ring mire when wearing each SCL, the NIKBUT program was used. The instrument immediately focused on the pigment of each SCL after starting the measurement automatically, and the sharpness of the ring mire in the image was confirmed.

Next, we investigated the relationship between the tear stability of two commercially available cosmetic SCLs with pigment in the lens surface: etafilcon A with polyvinylpyrrolidone (PVP), (1-Day Acuvue Moist Define; Johnson \& Johnson, New Brunswick, NJ, USA) and polymacon (I-lux innova dual color 1-Day; Innova vision Co. Ltd., Tokyo, Japan). The lens power was standardized at $-3.00 \mathrm{D}$. At first, we measured the tear film stability (NIBUT, NIKBUT-first, NIKBUT-average) without SCL. After the first randomly selected SCL was worn for 15 minutes, we measured NIBUT, NIKBUT-first, and NIKBUT-average over the SCL. Fifteen minutes after removing the first SCL, we again measured the tear film stability without SCL, and after the other SCL was worn for 15 minutes, we measured them over the SCL. The consistency of these parameters was investigated with and without SCL.

\section{Experiment 2}

In experiment 2, we actually evaluated the prelens tear film stability by two different methods using two types of silicone hydrogel daily disposable SCLs. In addition, We evaluated the wettability of SCLs using CA measurement in vitro. Additionally, we compared the functional VA, and subjective symptoms with two types of SCLs. The two commercially available daily disposable contact lenses were used in experiment 2: a moisture seal silicone hydrogel lens, samfilcon A (Ultra; Bausch and Lomb, Rochester, NY, USA) and a hydraclear 1 silicone hydrogel lens, narafilcon A (1-Day Acuvue True Eye; Johnson \& Johnson, New Brunswick, NJ, USA). Each SCL was available in powers ranging from $-0.50 \mathrm{D}$ to $-6.00 \mathrm{D}$. The subjects wore SCLs in both eyes, but measurements were performed only in the right eye. Examinations were conducted in each subject over a period of 3 days. On the first day, slit lamp assessment was performed to evaluate whether the subjects have dry eye and then we confirmed whether the SCLs used in this study fit each subject properly. Subjects wore SCLs randomly from the second to the third day. A break of at least 2 days was provided between each visit. Subjective symptoms, functional visual acuity, and the prelens tear film stability, ie, the NIBUT, NIKBUT-first, NIKBUTaverage, and TMH were measured at each visit after 8 hours of wear.

\section{Statistical Analysis}

Pearson's correlation coefficient was used to assess the correlations between the two tear film stability parameters. Bland Altman plot analysis was performed for calculating the difference and agreement between the measurements of the two instruments. For comparing the parameters when the subjects were wearing the two SCL types, paired $t$-test was used. $\mathrm{P} \leqq 0.05$ was considered significant. Prevalence of the BUPs was analyzed by chi-square test. Data and sample size were analyzed using JMP version 11 (SAS Institute, Inc., Cary, NC, USA).

\section{Results}

\section{Experiment I}

AS-OCT showed the location of the pigment in the front (Figure 1A) or back (Figure 1D) of the SCL. As recorded by the Keratograph, although the pigment located in the front of the SCL blurred when focus was on the ring mire (Figure 1B), the ring mire blurred when the pigment was focused (Figure 1C). In contrast, the ring mire was clear after focusing at the pigment located at the back of the SCL (Figure 1E). However, we could not focus on the surface of the SCL because of the absence of a target, such as a pigment (Figure 1F). Keratograph automatically begins measuring NIKBUT after focusing the clear ring mire. Thus, the focus of the ring mire recorded by the Keratograph while the subject was wearing the SCL, could be at the back of the SCL or the corneal surface but not the lens surface.

The NIBUT without SCL was significantly correlated with the NIKBUT-first without SCL ( $\mathrm{r}=0.445, \mathrm{P}=0.0488$, Pearson's correlation coefficients) (Figure 2A), but not with the NIKBUT-average without SCL (Figure 2B). The value of bias between the NIBUT and the NIKBUT-first and average without SCL were -1.20 and 5.44 seconds, respectively (Figure 2C and D). The value of limit of agreement (LOA) between these values ranged from -7.31 to 4.92 and -5.47 to 15.85 seconds, respectively (Figure 2C and D).

The NIBUT with SCL was not significantly correlated with the NIKBUT first and average with SCL (Figure 3A 

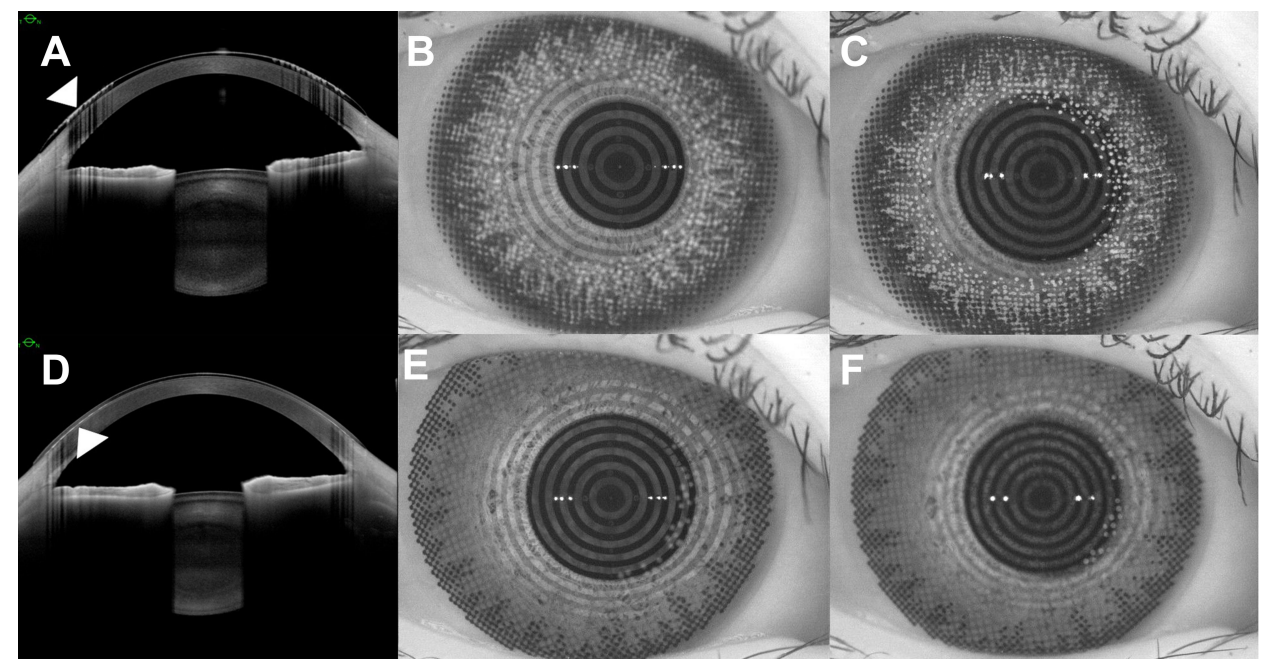

Figure I Arrow indicates the location of the pigment in the SCL front or back surface by anterior segment optical coherence tomography (A and $\mathbf{D})$, and photos of ring mire by the keratograph (B, C, E and F). (B) When we focus on the ring mire, pigment located at the front surface of the SCL blurs. (C) When we focus on the pigment, the ring mire blurs. (E) When we focus on the pigment located at the back surface of the SCL, the ring mire is also clear. (F) We do not focus on the lens surface of SCL because of absence of target.

Abbreviation: SCL, soft contact lens.
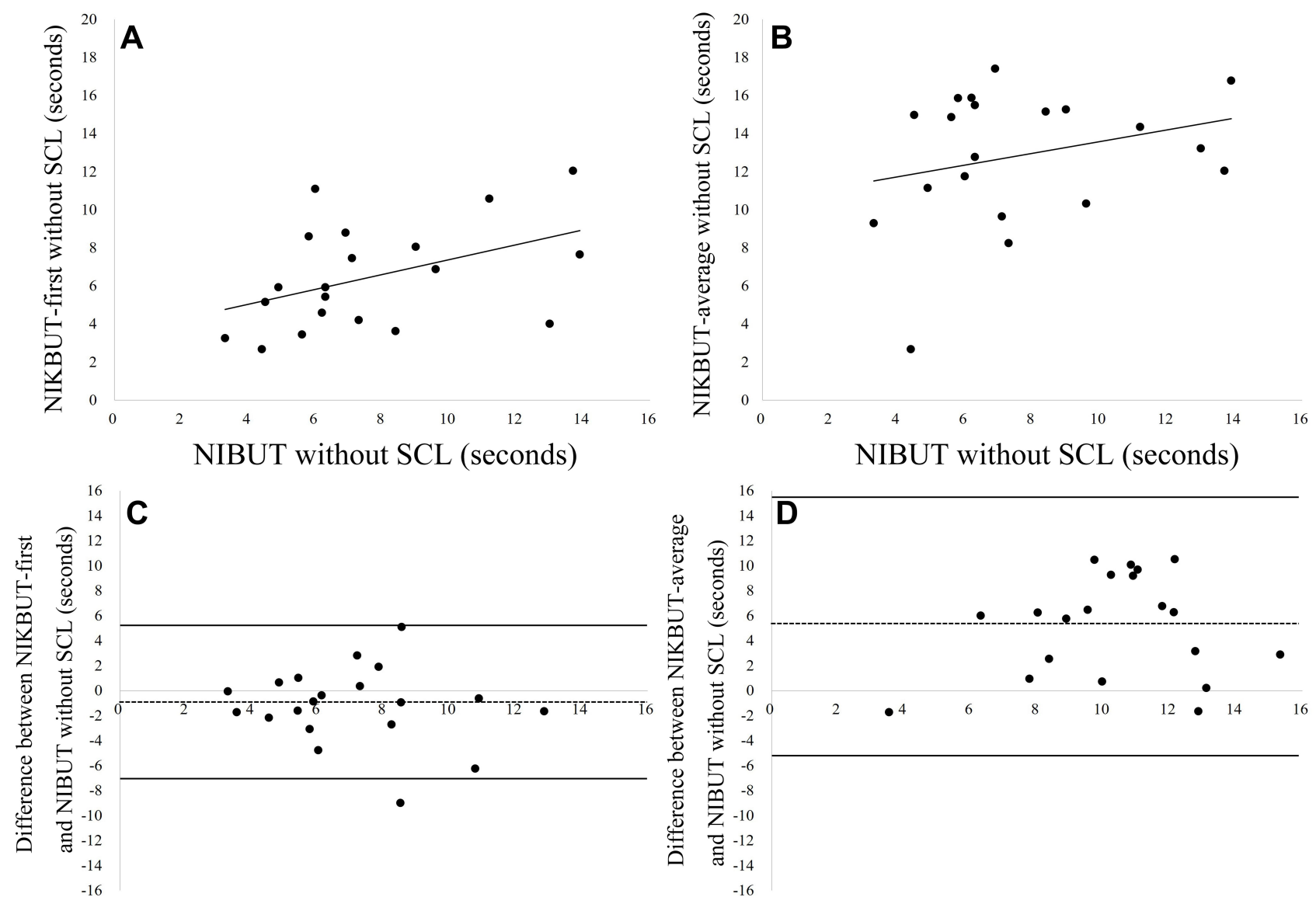

Average of NIKBUT-first and NIBUTwithout SCL (seconds)

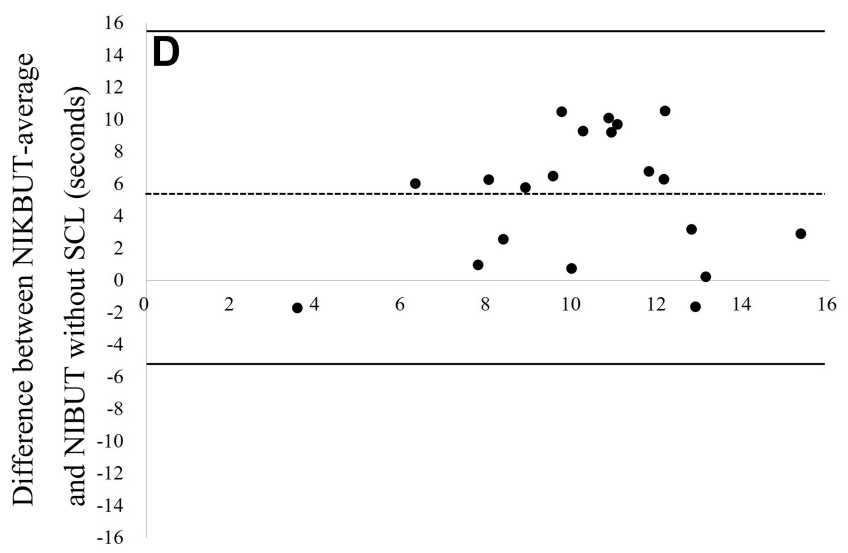

Average of NIKBUT-average and NIBUT without SCL (seconds)

Figure 2 Correlation between the NIBUT and (A) NIKBUT-first and (B) -average without SCL. Positive correlations are seen between the NIBUT and NIKBUT-first without SCL $(r=0.445, P=0.0488$, Pearson's correlation coefficients). Bland-Altman plot represented the agreement between the NIBUT and (C) NIKBUT-first and (D) NIKBUT-average without SCL. The solid line shows the upper and lower limits of agreement between the two parameters. The dotted line represents the mean differences between the two parameters. Abbreviations: NIBUT, noninvasive interferometry break up time; NIKBUT, noninvasive Keratograph break up time. 

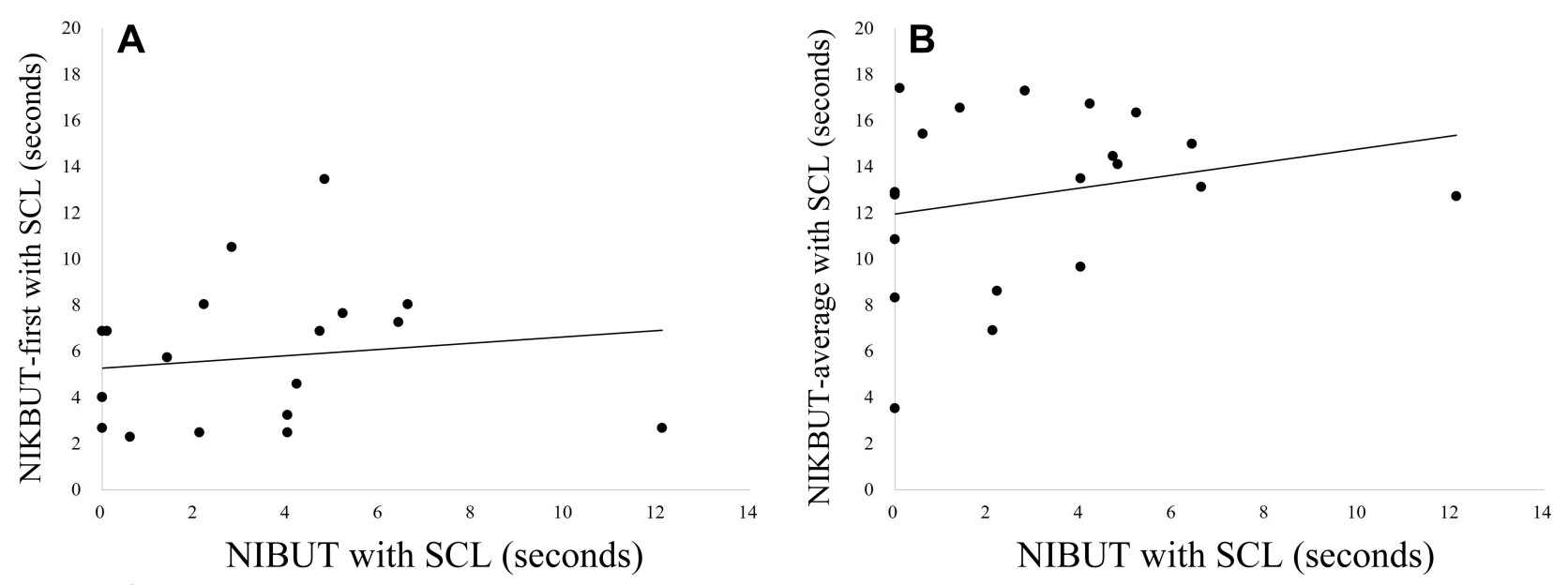

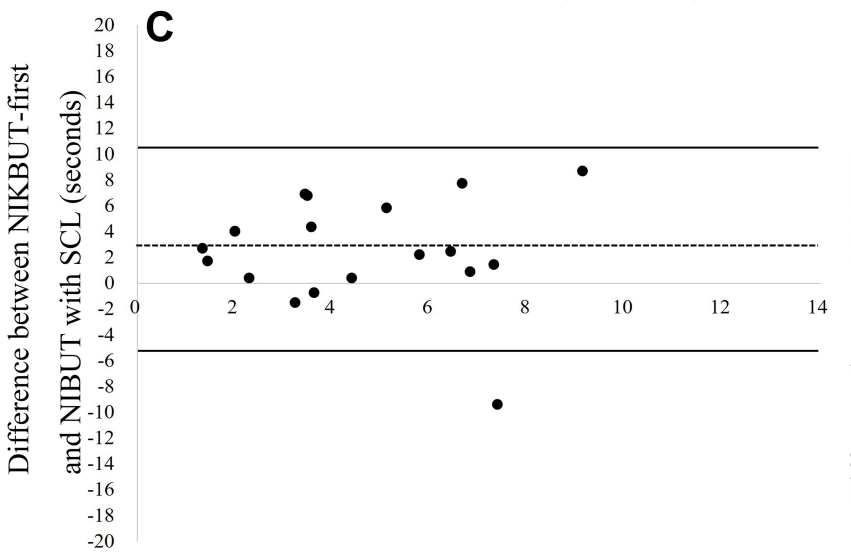

Average of NIKBUT-first and NIBUT with SCL (seconds)

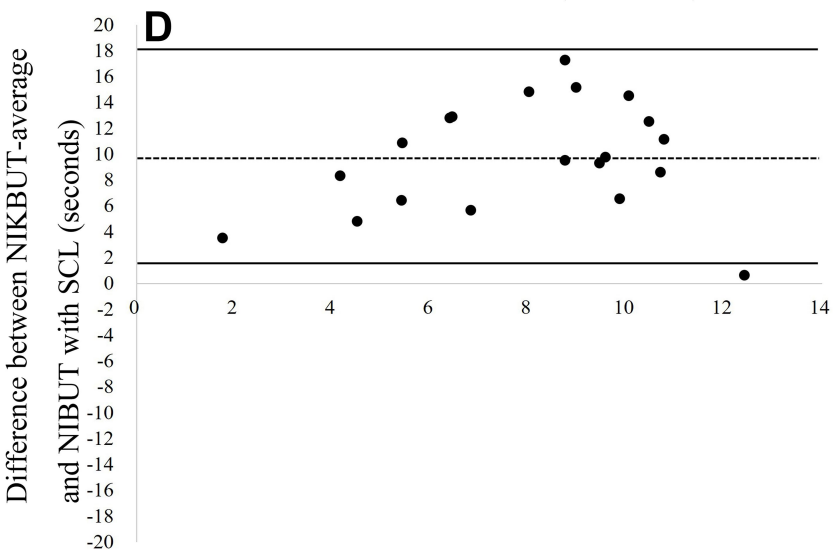

Average of NIKBUT-average and NIBUT with SCL (seconds)

Figure 3 Correlation between the NIBUT and (A) NIKBUT-first and (B) -average with SCL. The NIBUT was not significantly correlated with the NIKBUT-first and average. Bland-Altman plot represented the agreement between the NIBUT and (C) NIKBUT-first and (D) NIKBUT-average with SCL. The continuous line shows the upper and lower limits of agreement between the two parameters. The continuous line represents the mean differences between the two parameters.

Abbreviations: NIBUT, noninvasive interferometry break up time; NIKBUT, noninvasive Keratograph break up time.

and B). The value of bias between the NIBUT and the NIKBUT-first and average with SCL were 2.63 and 9.75 seconds, respectively (Figure $3 \mathrm{C}$ and $\mathrm{D}$ ). The value of LOA between these values ranged from -5.23 to 10.49 and 1.30 to 18.20 seconds, respectively (Figure $3 \mathrm{C}$ and D).

\section{Experiment 2}

The CA between saline and the SCL surface in samfilcon A and narafilcon A was $7.80 \pm 0.29$ and $8.52 \pm 0.25$, respectively (Table 1). The CA value of samfilcon A was significantly lower than that of narafilcon $\mathrm{A}(\mathrm{P}=0.0030$, Student's $t$-test $)$. The

Table I Comparison of Clinical Parameters Between the SCLs

\begin{tabular}{|l|l|l|l|}
\hline & Narafilcon A (10 Eyes) & Samfilcon A (10 Eyes) & P value \\
\hline CA (degree) & $8.52 \pm 0.25(8.36-8.67)$ & $7.80 \pm 0.29(7.62-7.97)$ & 0.0030 \\
TMH (mm) & $0.13 \pm 0.04(0.10-0.15)$ & $0.14 \pm 0.03(0.12-0.15)$ & 0.7655 \\
J-CLDEQ-8 & $2.40 \pm 4.12(-0.15-4.95)$ & $4.40 \pm 4.33(1.73-7.06)$ & 0.2208 \\
CDVA (log MAR) & $-0.05 \pm 0.10(-0.11-0.01)$ & $-0.04 \pm 0.07(-0.08-0.00)$ & 0.2633 \\
FVA (log MAR) & $0.01 \pm 0.03(0.00-0.03)$ & $0.00 \pm 0.02(-0.01-0.01)$ & 0.4224 \\
VMR (\%) & $0.98 \pm 0.03(0.96-0.99)$ & $0.97 \pm 0.04(0.94-0.99)$ & 0.4537 \\
Blink Rate (frequency/minute) & $17.90 \pm 10.12(11.62-24.17)$ & $21.20 \pm 6.92(16.91-25.48)$ & 0.2472 \\
\hline
\end{tabular}

Notes: The data are expressed as the average \pm deviation ( $95 \%$ confidence interval). Compared with narafilcon A, the CA differ significantly in the samfilcon A. Abbreviations: log MAR, logarithm of the minimum angle of resolution; CA, contact angle; TMH, tear meniscus height; J-CLDEQ-8, Japanese version of the 8-item contact lens dry eye questionnaire; CDVA, corrected distance visual acuity; FVA, functional visual acuity; VMR, visual maintenance rate. 
NIBUT with samfilcon A and narafilcon A was $4.52 \pm 2.43$ and $2.77 \pm 0.82$ seconds, respectively (Figure 4A). The NIBUT with samfilcon A was significantly longer than that of narafilcon A $(\mathrm{P}=0.0315$, paired $t$-test $)$. The NIKBUT-first with samfilcon $\mathrm{A}$ and narafilcon $\mathrm{A}$ was $8.14 \pm 6.81$ and $11.29 \pm 7.39$ seconds, respectively (Figure 4B). The NIKBUT-average with samfilcon A and narafilcon was $13.08 \pm 6.41$ and $16.35 \pm 4.45$ seconds, respectively (Figure 4C). The NIKBUT-first and average with SCL did not have significant difference between the two SCL types. The BUPs were classified to aqueous deficient type, decreased wettability type, and increased evaporation type. The rate of aqueous deficient type in samfilcon A and narafilcon A was $30 \%$ and $70 \%$, respectively. The rate of decreased wettability type was $10 \%$ and $30 \%$, respectively. The rate of increased evaporation type was $60 \%$ and $0 \%$, respectively. The rates of these types of BUPs for samfilcon $\mathrm{A}$ and narafilcon A showed significantly different trend $(\mathrm{P}=0.0041$, chi-square test). Table 1 shows the TMH, FVA, VMR, blink rate and subjective symptoms, which were not significantly different.

\section{Discussion}

To diagnose CLD, we need to understand prelens tear film stability. Over time, prelens tear film stability decreases and CLD worsens., ${ }^{7,33}$ Guillon et al reported that prelens tear film stability measured by interferometry in symptomatic subjects was less than that of asymptomatic subjects. $^{12}$ In experiment 1 , we found the ring mire of the NIKBUT program focused at the postlens/ corneal surface but not at the prelens surface. Although the NIKBUT-first without SCL was significantly correlated with the NIBUT without SCL, NIKBUT parameters with SCL showed no significant correlation with the NIBUT with SCL. In experiment 2, we compared the tear film stability of two daily disposable SCLs. The NIKBUT-first and NIKBUT-average were not significantly different, while the NIBUT was significantly different between SCLs. This implies that the NIKBUT program installed in the keratograph may not reflect to the prelens tear film during SCL wearing.

We attempted to evaluate the prelens tear film stability of the SCL surface by focusing on the pigment on the SCL surface in experiment 1 . However, despite the focus on the SCL surface, we could not detect the disturbance of the ring mire. Muller et al attempted measuring tear film stability using NIK-DUT, which was a method based on the conventional NIKBUT technique. ${ }^{34}$ NIK-DUT was recorded from the blink until the appearance of the first distortion of the ring mire by using a stopwatch and not automatic detection. They concluded that although NIKDUT did not differ significantly among three types of silicone hydrogel SCLs, their subjective wettability grades differed significantly. ${ }^{34}$ Considering the NIKBUT in vivo method did not find any significant difference among commercially available SCLs, ${ }^{25,26}$ it may assess the tear film stability on the cornea under the SCL.

We evaluated the tear film stability between two commercially available daily disposable SCLs using NIBUT, NIKBUT-first, and NIKBUT-average values. The NIBUT with samfilcon A was significantly longer than that for narafilcon A, but the NIKBUT-first and NIKBUT-average values were not significantly different between the two types of SCLs. Since the NIKBUT program may reflect tear film stability at the postlens or the corneal surface, tear stability between the lens and the cornea might not have differences between SCLs. The NIBUT values indicated that the prelens tear film stability of samfilcon A was better than that of narafilcon A. Results in vivo was consistent with data of CA in vitro. According to the type of BUPs, most of narafilcon A showed the aqueous deficient type, followed by the decreased wettability type. The
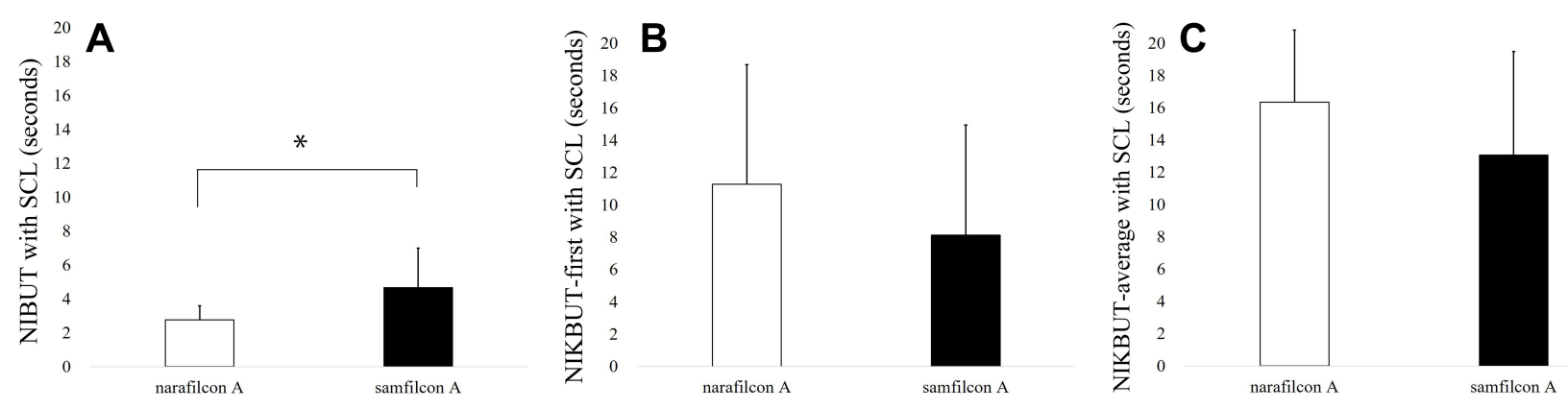

Figure 4 The comparison of tear film stability between SCLs using (A) NIBUT, (B) NIKBUT-first, and (C) NIKBUT-average. (A) Samfilcon A had significantly (*P<0.0I, paired $t$-test) longer NIBUT than narafilcon A. (B and $\mathbf{C}$ ) NIKBUT-first and average did not have significant difference between the SCLs. 
increased evaporation type accounted for the majority of all the BUPs in samfilcon A. It also implicates that samfilcon A could show the best wettability on the prelens surface among the tested SCLs.

Prelens tear film stability can be determined by various factors, including the tear volume ${ }^{11,13,35}$ and wettability ${ }^{36}$ of SCL materials. The wettability of SCL surface can be evaluated by contact angle in vitro. ${ }^{16,29} \mathrm{CA}$ may vary depending upon the SCL material, ${ }^{16}$ tear component, ${ }^{37}$ and tear supplements. ${ }^{29} \mathrm{TMH}$ representing the tear volume reduced while wearing the SCL compared to the TMH before wearing the SCL. ${ }^{38}$ Chen et al reported that lower the tear volume with SCLs, higher the subjective symptoms. ${ }^{38}$ In our study, the TMH was not significantly different between SCLs. Thus, the wettability of the SCL material, rather than the tear volume could determine tear film stability. Samfilcon A contains a large amount of PVP, which is known to improve wettability with the moisture seal technology. Hoteling et al reported that when the amount of PVP was investigated using gas chromatography with a flame ionization detector analysis, samfilcon A contained fourfold greater PVP than senofilcon A. ${ }^{39}$ Schafer et al reported that samfilcon A demonstrated a more wettable surface after blinking, compared to that of senofilcon A. ${ }^{40}$ In our study, the value of the CA with samfilcon A was significantly lower than that of narafilcon A, indicating that samfilcon A had more wetting material than narafilcon A. This study is the first to evaluate the tear volume and prelens tear film stability in vivo, and the wettability of SCL materials in vitro. We found that the prelens tear film stability changed depending on the wettability of the SCL material when the tear volume was the same.

Subjective symptoms and the FVA, VMR, and blink rate were not significantly different although the tear film stability was among the SCLs. The subjective symptoms may not only be related to the tear film stability ${ }^{12}$ and tear volume $^{13}$ but also lid wiper epitheliopathy. ${ }^{41}$ We did not assess these parameters because we aimed to investigate the tear film stability between SCLs. Koh et al reported that per J-CLDEQ-8, subjects with cutoff score of less than 11 points feel satisfied. ${ }^{32}$ In this study, almost all subjects obtained less than 11 . We recruited normal subjects who did not have any subjective symptoms. Subjects with CLD may lead to a difference in results among SCLs.

There were some limitations of the study. First, the sample size was increased to 20 by making it a crossover trial, and to eliminate inter-individual differences, we recruited
SCL wears without CLD. However, the sample size was too low. We will recruit a larger sample size, including asymptomatic and symptomatic subjects and investigate the association between CLD and various parameters, such as the tear film stability, tear volume, LWE, and morphology of the meibomian glands in the future. Second, NIKBUT provides clinically useful information by automatically analyzing the points of tear break over time, but does not focus on the SCL surface. In future, we would like to investigate the tear film stability with SCL using a NIKBUT program that can focus on the SCL surface.

In conclusion, we compared the tear film stability with SCL using interferometry and videokeratoscopy, and found that interferometry was more suitable to detect the prelens tear stability. The tear film stability was different among two types of SCLs. It was suggested that when the tear volume was same, the prelens tear film stability could change depending on the wettability of the SCL material.

\section{Abbreviations}

SCL, soft contact lens; NIBUT, noninvasive interferometry break up time; TFOS, Tear Film and Ocular Surface; CLD, Contact Lens Discomfort; CA, contact angle; BUP, breakup pattern; TBUT, tear breakup time; TMH, tear meniscus height; NIKBUT, noninvasive Keratograph break up time; UMIN, University Hospital Medical Information Network; AB, area break; LB, line break; TALB, thin aqueous layer break; SB, spot break; DB, dimple break; RB, random break.

\section{Ethical Approval and Informed Consent}

This study was approved by the Institutional Review Board of River Side Clinic and adhered to the tenets of the Declaration of Helsinki. Informed consent was obtained from the patients after explaining the nature and possible consequences of the study.

\section{Acknowledgments}

We would like to thank Editage (www.editage.com) for English proofread our English writing.

\section{Author Contributions}

All authors made substantial contributions to conception and design, acquisition of data, or analysis and interpretation of data; too part in drafting the article or revising it critically for 
important intellectual content; agreed to submit to the current journal; gave final approval of the version to be published; and agree to be accountable for all aspects of the work.

\section{Disclosure}

This was a contract study sponsored by Bausch + Lomb Japan. (Tokyo, Japan). Takashi Suzuki reports grants from Menicon, Ltd. and ALCON JAPAN, Ltd., outside the submitted work. Yuichi Hori reports grants from ALCON JAPAN, Ltd., HOYA Corporation, and Menicon, Ltd., outside the submitted work. The authors report no other potential conflicts of interest for this work.

\section{References}

1. Dumbleton K, Caffery B, Dogru M, et al. The TFOS international workshop on contact lens discomfort: report of the subcommittee on epidemiology. Invest Ophthalmol Vis Sci. 2013;54(11):20-36. doi:10.1167/iovs.13-13125

2. Taneri S, Oehler S, MacRae S, Dick HB. Influence of a therapeutic soft contact lens on epithelial healing, visual recovery, haze, and pain after photorefractive keratectomy. Eye Contact Lens. 2018;44(Suppl 1):S38-S43. doi:10.1097/ICL.0000000000000311

3. Moezzi AM, Varikooty J, Schulze M, et al. Corneal swelling with cosmetic etafilcon A lenses versus no lens wear. Optom Vis Sci. 2016;93(6):619-628. doi:10.1097/OPX.0000000000000840

4. Muniesa MJ, Ezpeleta J, Benitez I. Fluctuations of the intraocular pressure in medically versus surgically treated glaucoma patients by a contact lens sensor. Am J Ophthalmol. 2019;203:1-11. doi:10.1016/ j.ajo.2019.02.003

5. Teo L, Lim L, Tan DTH, Chan T, Jap A, Ming LH. A survey of contact lens complications in Singapore. Eye Contact Lens. 2011;37 (1):16-19. doi:10.1097/ICL.0b013e3182048f99

6. Chalmers R, Long B, Dillehay S, Begley C. Improving contact-lens related dryness symptoms with silicone hydrogel lenses. Optom Vis Sci. 2008;85(8):778-784. doi:10.1097/OPX.0b013e318181a90d

7. Subbaraman LN, Glasier M, Varikooty J, et al. Protein deposition and clinical symptoms in daily wear of etafilcon lenses. Optom Vis Sci. 2012;89(10):1450-1459. doi:10.1097/OPX.0b013e318269e583

8. Nichols KK, Redfern RL, Jacob JT, et al. The TFOS international workshop on contact lens discomfort: report of the definition and classification subcommittee. Invest Ophthalmol Vis Sci. 2013;54(11): TFOS20-36. doi:10.1167/iovs.13-13074

9. Gumus K, Crockett $\mathrm{CH}$, Rao K, et al. Noninvasive assessment of tear stability with the tear stability analysis system in tear dysfunction patients. Invest Ophthalmol Vis Sci. 2011;52(1):456-461. doi:10.1167/iovs.105292

10. Szczesna-Iskander DH, Iskander DR, Read SA, Alonso-Caneiro D. Noninvasive in vivo assessment of soft contact lens type on tear film surface quality. Invest Ophthalmol Vis Sci. 2012;53(1):525-531. doi:10.1167/iovs.11-8257

11. Maruyama K, Yokoi N, Takamata A, Kinoshita S. Effect of environmental conditions on tear dynamics in soft contact lens wearers. Invest Ophthalmol Vis Sci. 2004;45(8):2563-2568. doi:10.1167/iovs.03-1185

12. Guillon M, Dumbleton KA, Theodoratos P, et al. Association between contact lens discomfort and prelens tear film kinetics. Optom Vis Sci. 2016;93(8):881-891. doi:10.1097/OPX.0000000000000866

13. Itokawa T, Okajima Y, Suzuki T, et al. Association between ocular surface temperature and tear film stability in soft contact lens wearers. Invest Ophthalmol Vis Sci. 2018;59(2):771-775. doi:10.1167/iovs.17-2 3173
14. Siddireddy JS, Vijay AK, Tan J, Willcox M. The eyelids and tear film in contact lens discomfort. Cont Lens Anterior Eye. 2018;41 (2):144-153. doi:10.1016/j.clae.2017.10.004

15. Purslow C, Wolffsohn JS, Santodomingo-Rubido J. The effect of contact lens wear on dynamic ocular surface temperature. Cont Lens Anterior Eye. 2005;28(1):29-36. doi:10.1016/j.clae.2004.10.001

16. Efimov P, Yokoi N, Peev N, Georgiev GA. Impact of air exposure time on the water contact angles of daily disposable silicone hydrogels. Curr Int J Mol Sci. 2019;20(6):1313. doi:10.3390/ ijms20061313

17. Kaido M, Kawashima M, Ishida R, Tsubota K. Tear film dynamics of soft contact lens- induced dry eye. Curr Eye Res. 2020;45 (7):782-788. doi:10.1080/02713683.2019.1700530

18. Yokoi N, Georgiev GA. Tear- film- oriented diagnosis for dry eye. Jpn J Ophthalmol. 2019;63(2):127-136. doi:10.1007/s10384-018-00645-4

19. Yokoi N, Sakai R, Georgiev GA, Kato H, Sotozono C, Kinoshita S. Impact of soft contact lens wear on tear film breakup patterns, meniscus tear volume, and tear film stability. Invest Ophthalmol Vis Sci. 2018;59:E-Abstract1751.

20. Mao X, Savini G, Zhuo Z, et al. Repeatability, reproducibility, and agreement of corneal power measurements obtained with a new corneal topographer. J Cataract Refract Surg. 2013;39(10):15 61-1569. doi:10.1016/j.jcrs.2013.04.029

21. Jiang Y, Ye H, Xu J, Lu X. Noninvasive keratograph assessment of tear film break-up time and location in patients with age-related cataracts and dry eye syndrome. J Int Med Res. 2014;42 (2):494-502. doi:10.1177/0300060513504701

22. Menzies KL, Srinivasan S, Prokopich CL, Jones L. Infrared imaging of meibomian glands and evaluation of the lipid layer in sjogren's syndrome patients and nondry eye controls. Invest Ophthalmol Vis Sci. 2015;56(2):836-841. doi:10.1167/iovs.14-13864

23. Wu S, Hong J, Tian L, Cui X, Sun X, Xu J. Assessment of bulbar redness with a newly developed keratography. Optom Vis Sci. 2015;92(8):892-899. doi:10.1097/OPX.0000000000000643

24. Gracia-Montero M, Rico-Del-Viejo L, Lorente-Velazquez A, Martinez-Alberquilla I, Hernandez-Verdejo JL, Madrid-Costa D. Repeatability of noninvasive keratograph $5 \mathrm{M}$ measurements associated with contact lens wear. Eye Contact Lens. 2019;45 (6):377-381. doi:10.1097/ICL.0000000000000596

25. Gracia-Montero M, Rico-Del-Viejo L, Llorens-Quintana C, et al. Randomized crossover trial of silicone hydrogel contact lenses. Cont Lens Anterior Eye. 2019;42(5):475-481. doi:10.1016/j.clae.2018.12.006

26. Mousavi M, Jesus DA, Garaszczuk IK, Szczesna-Iskander DH, Iskander DR. The utility of measuring tear film break-up time for prescribing contact lenses. Cont Lens Anterior Eye. 2018;41 (1):105-109. doi:10.1016/j.clae.2017.08.003

27. Tsubota K, Yokoi N, Shimazaki J, et al. New perspectives on dry eye definition and diagnosis: a consensus report by the Asia dry eye society. Ocul Surf. 2017;15(1):65-76. doi:10.1016/j.jtos.2016.09.003

28. Itokawa $\mathrm{T}$, Iwashita $\mathrm{H}$, Okajima $\mathrm{Y}$, et al. Changes in ocular surface temperature after instillation of $3 \%$ diquafosol ophthalmic solution over soft contact lenses. J Jpn CL Soc. 2018;60:91-95.

29. Iwashita H, Itokawa T, Suzuki T, Okajima Y, Kakisu K, Hori Y. Evaluation of in vitro wettability of soft contact lenses using tear supplements. Eye Contact Lens. 2020;Publish Ahead of Print. doi:10.1097/ICL.0000000000000698.

30. Kaido M, Dogru M, Ishida R, Tsubota K. Concept of functional visual acuity and its applications. Cornea. 2007;26(9 Suppl 1):S29S35. doi:10.1097/ICO.0b013e31812f6913

31. Kaido M. Functional visual acuity. Invest Ophthalmol Vis Sci. 2018;59(14):DES29-DES35. doi:10.1167/iovs.17-23721

32. Koh S, Chalmers R, Kabata D, Shintani A, Nishida K. Translation and validation of the 8-item contact lens dry eye questionnaire (CLDEQ-8) among Japanese soft contact lens wearers: J-CLDEQ-8. Cont Lens Anterior Eye. 2019;42(5):533-539. doi:10.1016/j.clae.20 19.03 .002 
33. Papas E, Tilia D, McNally J, Jara PL. Ocular discomfort responses after short periods of contact lens wear. Optom Vis Sci. 2015;92 (6):665-670. doi:10.1097/OPX.0000000000000607

34. Muller C, Marx S, Wittekind J, Sickenberger W. Subjective comparison of prelens tear film stability of daily disposable contact lenses using ring mire. Clin Optom. 2020;28(12):17-26. doi:10.2147/OPTO. S235167

35. Yokoi N, Yamada H, Mizukusa Y, et al. Rheology of tear film lipid layer spread in normal and aqueous tear-deficient dry eyes. Invest Ophthalmol Vis Sci. 2008;49(12):5319-5324. doi:10.1167/iovs.071407

36. Read ML, Morgan PB, Maldonado-Codina C. Measurement errors related to contact angle analysis of hydrogel and silicone hydrogel contact lenses. J Biomed Mater Res B Appl Biomater. 2009;91 (2):662-668. doi:10.1002/jbm.b.31442

37. Lorentz H, Rogers R, Jones L. The impact of lipid on contact angle wettability. Optom Vis Sci. 2007;84(10):946-953. doi:10.1097/ OPX.0b013e318157a6c1
38. Chen Q, Wang J, Shen M, et al. Tear menisci and ocular discomfort during daily contact lens wear in symptomatic wearers. Invest Ophthalmol Vis Sci. 2011;52(5):2175-2180. doi:10.1167/iovs.105780

39. Hoteling AJ, Nichols WF, Harmon PS, et al. Characterization and quantitation of PVP content in a silicone hydrogel contact lens produced by dual-phase polymerization processing. J Biomed Mater Res B Appl Biomater. 2018;106(3):1064-1072. doi:10.1002/jbm. b.33904

40. Schafer J, Reindel W, Steffen R, et al. Use of a novel extended blink test to evaluate the performance of two polyvinylpyrrolidone-containing, silicone hydrogel contact lenses. Clin Ophthalmol. 2018;12:819-825. doi:10.2147/OPTH.S162233

41. Korb DR, Greiner JV, Herman JP, et al. Lid-wiper epitheliopathy and dry-eye symptoms in contact lens wearers. CLAO J. 2002;28 (4):211-216. doi:10.1097/01.ICL.0000029344.37847.5A
Clinical Ophthalmology

\section{Publish your work in this journal}

Clinical Ophthalmology is an international, peer-reviewed journal covering all subspecialties within ophthalmology. Key topics include: Optometry; Visual science; Pharmacology and drug therapy in eye diseases; Basic Sciences; Primary and Secondary eye care; Patient Safety and Quality of Care Improvements. This journal is indexed on PubMed

Submit your manuscript here: https://www.dovepress.com/clinical-ophthalmology-journal

\section{Dovepress}

Central and CAS, and is the official journal of The Society of Clinical Ophthalmology (SCO). The manuscript management system is completely online and includes a very quick and fair peer-review system, which is all easy to use. Visit http://www.dovepress.com/ testimonials.php to read real quotes from published authors. 